ISSN 0103-9954

\title{
DINÂMICA DA ESTRUTURA DA VEGETAÇÃO DO SUB-BOSQUE SOB INFLUÊNCIA DA EXPLORAÇÃO EM UMA FLORESTA DE TERRA FIRME NO MUNICÍPIO DE MOJU - PA ${ }^{1}$
}

\author{
DYNAMICS STRUCTURE OF THE UNDERSTOREY VEGETATION INFLUENCED BY LOGGING \\ IN A TERRA FIRME FOREST, IN THE MUNICIPALITY OF MOJU, PARÁ STATE
}

Fernanda da Silva Mendes ${ }^{2}$ Fernando Cristóvam da Silva Jardim ${ }^{3}$ João Olegário Pereira de Carvalho ${ }^{4}$ Deivison Venício Souza ${ }^{5}$ Clívia Bezerra Araújo ${ }^{6}$ Mariana Gomes de Oliveira ${ }^{7}$ Eduardo da Silva Leal ${ }^{8}$

\section{RESUMO}

Analisou-se a dinâmica da estrutura da vegetação do sub-bosque de uma floresta de terra firme sob a influência de clareiras durante 12 anos após a exploração florestal de impacto reduzido no município de Moju, Pará, na Amazônia Oriental, Brasil. Angiospermas de $10 \mathrm{~cm}$ de altura a $5 \mathrm{~cm}$ de DAP foram inventariadas em $468 \mathrm{~m}^{2}$ de amostras, em seis ocasiões (1998, 1999, 2000, 2001, 2007 e 2010), analisadas de acordo com as formas de vida (árvores, arbustos, lianas, ervas e palmeiras), considerando a abundância, a frequência, as categorias de tamanho $(\mathrm{CT} 1=10 \mathrm{~cm} \leq \mathrm{HT}<50 \mathrm{~cm}$; CT2 $=50 \mathrm{~cm} \leq \mathrm{HT} \leq 130 \mathrm{~cm}$; CT3 $=130 \mathrm{~cm}<$ HT e DAP $<2 \mathrm{~cm}$; e CT4 $=2 \mathrm{~cm} \leq \mathrm{DAP}<5 \mathrm{~cm}$ ) e a regeneração natural relativa. As famílias mais representativas foram Burseraceae, Marantaceae, Violaceae e Lecythidaceae em todas as ocasiões, enquanto as espécies mais importantes foram Monotagma sp1., Monotagma sp2., Rinorea guianensis, Lecythis idatimon e Protium pilosum. As formas de vida mais representativas foram, em ordem decrescente, árvores, ervas e lianas em todas as ocasiões. A dinâmica das diferentes formas de vida foi dependente da intensidade de luz. As clareiras possibilitaram o estabelecimento de regeneração de espécies comerciais e potencialmente comerciais, indicando que essas espécies não necessitam de condução para garantir o estoque para futuros cortes.

Palavras-chave: Amazônia Oriental; exploração de impacto reduzido; clareiras.

\section{ABSTRACT}

The structure dynamics of an understorey vegetation influenced by gaps caused by reduced impact logging was analyzed during 12 years in a terra firme rain forest in the municipality of Moju, Pará state, eastern Brazilian Amazon forest. Angiosperm individuals from $10 \mathrm{~cm}$ height up to $5 \mathrm{~cm}$ DBH were surveyed in a $468 \mathrm{~m}^{2}$ sample area in six occasions $(1998,1999,2000,2001,2007$ and 2010), they were analyzed considering their life-forms (trees, shrub, lianas, herbs and palms), abundance, frequency, size classes (CT1 $=10 \mathrm{~cm} \leq \mathrm{HT}<50 \mathrm{~cm} ; \mathrm{CT} 2=50 \mathrm{~cm} \leq \mathrm{HT} \leq 130 \mathrm{~cm} ; \mathrm{CT} 3=130 \mathrm{~cm}<\mathrm{HT}$ and DAP $<2 \mathrm{~cm} ;$ and $\mathrm{CT} 4=2 \mathrm{~cm}$ $\leq$ DAP $<5 \mathrm{~cm}$ ) and relative natural regeneration Burseraceae, Marantaceae, Violaceae and Lecythidaceae were the most important families in the study area in every occasions, while the most representative species

1. Parte da dissertação de mestrado da primeira autora apresentada ao Programa de Pós-graduação em Ciências Florestais da Universidade Federal Rural da Amazônia (PA).

2. Engenheira Florestal, MSc., Professora do Departamento de Tecnologia e Recursos Naturais, Universidade do Estado do Pará, CEP: 68.503-120, Marabá (PA).mendes.fsm@gmail.com

3. Engenheiro Florestal, Dr.., Professor do Instituto de Ciências Agrárias, Universidade Federal Rural da Amazônia, Caixa Postal 917, CEP 66077-530, Belém (PA). fernando.jardim@ufra.edu.br

4. Engenheiro Florestal, DPhil., Professor do Programa de Pós-graduação em Ciências Florestais, Universidade Federal Rural da Amazônia, Caixa Postal 917, CEP 66077-530, Belém (PA). olegario.carvalho@gmail.com

5. Engenheiro Florestal, MSc., Professor da Faculdade de Engenharia Florestal, Universidade Federal do Pará. CEP 68372040, Altamira (PA).deivisonvs@ufpa.br.

6. Engenheira Florestal,MSc.,AnalistaAmbiental IBAMA,CEP68464-000,Tucuruí(PA).clivia.araujo@hotmail.com

7. Engenheira Agrônoma, MSc., Professora do Instituto Federal do Pará. CEP 68502-120, Marabá (PA). marianaoliveirag22@hotmail.com

8. Engenheiro Florestal, MSc., Professor da Universidade Federal Rural da Amazônia, CEP 68700-030, Capanema (PA).edu_engflor@yahoo.com.br

Recebido para publicação em 4/04/2011 e aceito em 16/01/2012 
were Monotagma sp1., Monotagma sp2., Rinorea guianensis, Lecythis idatimon e Protium pilosum. The most frequent life forms were trees, herbs and lianas in all occasions. The dynamics of life forms depended on light intensity. The establishment of commercial and potentially commercial species was favored by gaps, suggesting that natural regeneration of those species do not need to be assisted for guaranteeing timber stocks for future harvests.

Keywords: Eastern Amazon forest; reduced impact logging; canopy gaps.

\section{INTRODUÇÃO}

A estrutura de uma comunidade vegetal é importante para o entendimento do ecossistema florestal e, consequentemente, para garantir o seu uso sustentável. A análise da estrutura requer o conhecimento de suas espécies, pois se baseia no relacionamento entre elas, levando em consideração as suas possíveis associações (PIRES-O'BRIEN e O'BRIEN, 1995).

Diversos trabalhos têm sido realizados na região amazônica em florestas de terra firme visando à obtenção de informações qualitativas e quantitativas, analisando principalmente a estrutura horizontal da comunidade arbórea (LIMA FILHO et al., 2002; ALVES e MIRANDA, 2008; OLIVEIRA et al., 2008, SILVA et al., 2008). O estudo da estrutura vertical é de fundamental importância para a determinação da estrutura nas florestas tropicais, quando o objetivo é subsidiar o manejo florestal (FINOL, 1971).

Dentro da estrutura florestal, os indivíduos jovens são considerados indicador da estrutura futura da comunidade, dependendo apenas de condições favoráveis para se desenvolverem (FELFILI et al.,2001). Para que uma população arbórea específica se mantenha numa determinada comunidade florestal, espera-se encontrar representatividade da espécie em todos os estratos da vegetação (SALLES e SCHIAVINI, 2007).

O sub-bosque forma um nicho ecológico de vital importância para o estabelecimento e desenvolvimento das espécies que irão constituir os demais estratos da floresta (JARDIM e HOSOKAWA, 1987; OLIVEIRA e AMARAL, 2005). Além disso, as espécies exclusivas do estrato inferior da comunidade vegetal possuem papel importante na composição da área, exercendo a função de cobertura do solo, além da contribuição ecológica nas mais variadas interações biológica com as espécies arbóreas (LIMA FILHO et al., 2002).

Com o intuito de contribuir para o conhecimento sobre estrutura da regeneração de espécies arbóreas e de comunidades de ervas, arbustos, pal- meiras e lianas, subsidiando a silvicultura de florestas tropicais, este trabalho teve como objetivo avaliar as mudanças ocorridas na estrutura da vegetação do sub-bosque, considerando as formas de vida, sob a influência das clareiras, durante o período de 12 anos de monitoramento após a exploração florestal.

\section{MATERIAL E MÉTODOS}

\section{Área de Estudo}

O estudo foi realizado em uma área de 200 ha, no Campo Experimental da Embrapa Amazônia Oriental, km 30 da Rodovia PA 150, no município de Moju, Pará. A vegetação é do tipo Floresta Tropical de Terra Firme ou Floresta Ombrófila Densa (VELOSO et al., 1991), com espécies arbóreas de grande porte, altura do dossel variando de 25 a $30 \mathrm{~m}$.

O tipo climático é Ami (quente e úmido), segundo a classificação de Köppen, com temperatura média anual oscilando de 25 a $27^{\circ} \mathrm{C}$; insolação mensal de $148,0 \mathrm{~h}$ a $275,8 \mathrm{~h}$, umidade relativa do ar em torno de $85 \%$ e precipitação anual de 2000 a $3000 \mathrm{~mm}$, com o período mais chuvoso de janeiro a junho. O solo predominante é latossolo amarelo, o relevo plano com pequenos desnivelamentos variando de $0 \%$ a $3 \%$. (SANTOS et al., 1985).

Foi realizada exploração florestal de impacto reduzido de acordo com a legislação vigente, no período de outubro-novembro de 1997 (SILVA et al., 2001). Foram colhidas 24 espécies, destacando-se Pseudopiptadenia suaveolens (Miq.) J. W. Grimes, Vouacapoua americana Aubl. e Manilkara huberi (Ducke) A Chev. (LOPES et al., 2001).

\section{Desenho do Experimento e Coleta de dados}

Foram selecionadas nove clareiras da exploração, considerando como tal a área da floresta com dossel descontínuo, aberta pela queda de árvores, limitada pelas copas periféricas. Suas áreas foram estimadas através da área do circulo, utilizando a média do diâmetro maior e seu diâmetro perpendi- 
cular. As áreas variaram de 231 a $748 \mathrm{~m}^{2}$, com média de $497 \mathrm{~m}^{2}$. Em cada clareira foram instaladas 13 parcelas permanentes quadradas de $2 \times 2 \mathrm{~m}$, dispostas conforme a Figura 1, perfazendo uma amostra de $468 \mathrm{~m}^{2}$.

Foi registrada e medida a altura total $(\mathrm{HT}) \mathrm{de}$ todas as angiospermas, compreendida de $\mathrm{HT} \geq 10 \mathrm{~cm}$ e DAP $<5 \mathrm{~cm}$. Foi coletado material botânico, quando possível com amostras férteis, para identificação no herbário. A listagem florística seguiu o sistema de classificação APG III (2009). Foram consideradas espécies, gêneros e famílias botânicas, cujos nomes científicos foram conferidos mediante consulta no "site" The International Plant Names Index (IPNI, 2010). Indivíduos não identificados foram contabilizados na amostra como Não Identificado (NI), sendo agrupados para facilitar as análises.

Foram realizadas quatro medições entre os anos de 1998 a 2001, iniciadas quatro meses após a exploração, no mês de março. Posteriormente, foi realizada a quinta medição em outubro de 2007 e a sexta e última medição em abril de 2010, totalizando seis medições.

\section{Análise de dados}

A classificação das formas de vida das espécies foi efetuada por observações em campo, de acor-
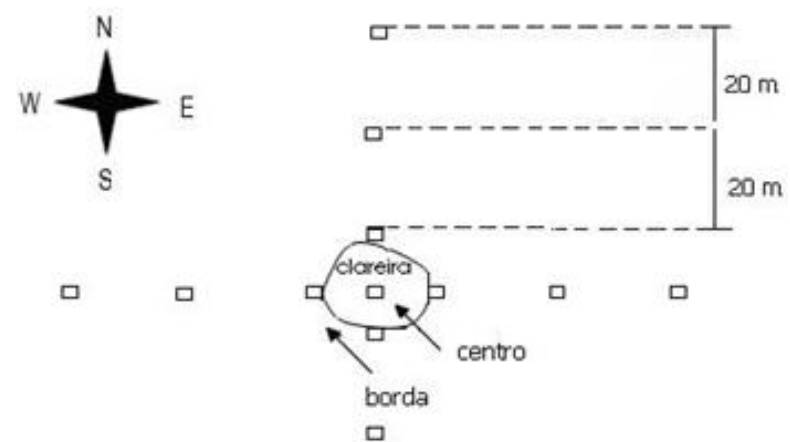

- Parcela $2 \times 2 \mathrm{~m}$

口

FIGURA 1: Disposição das parcelas em relação às clareiras de exploração florestal, no Campo Experimental da Embrapa Amazônia Oriental, no município de Moju - PA.

FIGURE 1: Lay out of the permanent sample plots with regard to logging gaps, at 'Embrapa Amazônia Oriental Experimental Station', in Moju municipality, Pará state. do com Santos et al. (2003) e Kozera et al. (2009): árvore (AR) - planta lenhosa que ramifica acima de $0,5 \mathrm{~m}$ de altura; arbusto (AB) - planta lignificada apenas na base, que ramifica até $0,5 \mathrm{~m}$ de altura; palmeira (PA) - espécies de Arecaceae; erva (ER) planta não lignificada; liana (LI) - planta escandente.

Foi considerada a estratificação da vegetação em quatro categorias de tamanho, de acordo com Jardim e Souza (1996), da seguinte forma: CT1 = $10 \mathrm{~cm} \leq \mathrm{HT}<50 \mathrm{~cm} ; \mathrm{CT} 2=50 \mathrm{~cm} \leq \mathrm{HT} \leq 130 \mathrm{~cm}$; $\mathrm{CT} 3=130 \mathrm{~cm}<$ HT e DAP $<2 \mathrm{~cm} ;$ CT $4=2 \mathrm{~cm} \leq$ $\mathrm{DAP}<5 \mathrm{~cm}$.

A análise estrutural da vegetação do sub-bosque foi realizada utilizando-se a metodologia sugerida por Finol (1971), amplamente empregada por vários autores como Jardim e Hosokawa (1986/87), Oliveira e Amaral (2005), Lima Filho et al. (2002), entre outros.

A estrutura horizontal é representada pelos parâmetros que estimam a ocupação do solo pela espécie no plano horizontal da floresta (JARDIM e HOSOKAWA, 1986/87). Para tal, foram calculados os valores absolutos e relativos de frequência e abundância (HOSOKAWA, 1986). Não foi avaliada a dominância devido à maioria dos indivíduos serem menores que 1,30 de altura ou possuírem diâmetro insignificante em relação à estrutura diamétrica da floresta.

Para analisar a estrutura vertical da vegetação do sub-bosque, que considera a ocupação da floresta por estratos em relação à altura, foi determinada a Categoria de Tamanho Relativa da Regeneração Natural, dada pela fórmula (FINOL, 1971):

$$
C T \%_{s p}=\frac{C T_{s p}}{\sum C T} \times 100
$$

onde: $C T_{s p}=\left[\left(\mathrm{n}_{1} \mathrm{~N}_{1}+\ldots+\mathrm{n}_{4} \mathrm{~N}_{4}\right) \div \mathrm{N}\right] \times 100$, $C T_{s p}=$ Categoria de tamanho de cada espécies; $n_{1, \ldots, 4}$ $=$ número de indivíduos de cada espécie, respectivamente nas categorias de tamanho 1 a $4 ; N_{I_{4}}=$ número total de indivíduos nas categorias de tamanho 1 a $4 ; N=$ número total de indivíduos da amostragem.

As espécies foram hierarquizadas em importância pela Regeneração Natural Relativa, que é obtida pela soma dos valores relativos de abundância, frequência e categoria de tamanho da regeneração natural (FINOL, 1971). Os parâmetros estimados para a abordagem da estrutura vertical e horizontal foram analisados em todas as medições e relacionados no decorrer dos anos. 
Testes de normalidade e homocedasticidade de variâncias foram realizados a fim avaliar as suposições de normalidade e variâncias homogêneas exigidas pelo teste paramétrico. Para o teste de normalidade foi utilizado o teste de KolmogorovSmirnov baseado no critério de $p>0,05$. Para o teste de homocedasticidade de variâncias foi adotado o teste de Levene. Como os dados apresentaram distribuição normal, foi utilizada a análise de variância da abundância, frequência e categoria de tamanho absolutas dos anos. Para resultado significativo $(\mathrm{p}<$ $0,05)$ realizou-se o Teste de Tukey conforme descrito por Pimentel-Gomes e Garcia (2002).

Foi calculado o coeficiente de correlação não paramétrica ( $r$ de Spearman) para os valores absolutos por espécies de Abundância, Frequência e Categoria de Tamanho nos diferentes anos amostrados para verificar a interação entre os parâmetros de estrutura estimados.

\section{RESULTADOS E DISCUSSÃO}

Os valores médios absolutos de abundância (AA), categoria de tamanho (CT) e frequência (FR) nos diferentes anos estão detalhados na Figura 2. Todos os parâmetros da comunidade foram crescentes até 2000 e decrescentes nos anos posteriores, diferença ratificada pela análise de variância (AA: $\mathrm{F}=23,76, \mathrm{p}$-valor $<0,001 ; \mathrm{CT}: \mathrm{F}=20,52$, $\mathrm{p}$-valor $<0,001$; e FR: $F=16,917$, p-valor $<0,001)$ e pelo teste de Tukey.

Esse adensamento verificado até o terceiro ano pode ser explicado pela formação de clareiras. No início do estudo, em março de 1998, quatro meses após a exploração, os efeitos da abertura das clareiras, tais como disponibilidade de luz, redução da umidade do ar, aumento da temperatura da área, propriedades do solo (BLOOR e GRUBB 2003; WHITMORE 1984) ainda não eram evidentes na vegetação, e foram mais marcantes no primeiro e segundo ano após a abertura do dossel, com o intenso recrutamento de indivíduos.

Os níveis de abertura do dossel da área estudada foram analisados por Lopes et al. (2001) e constataram que em 1997, após a exploração, cerca de $20 \%$ da área apresentava no mínimo intensa luz lateral nas copas ou aberturas superiores; já em 1998, devido a perturbações naturais e consequências recorrentes da exploração, esse nível subiu para cerca de $40 \%$, o que ratifica a influência da luz na área estudada.

O coeficiente de correlação $r$ de Spearman foi de $0,968,0,958$ e 0,927 entre abundância e frequência, abundância e categoria de tamanho e frequência e categoria de tamanho, respectivamente (p-valor $<0,01 \mathrm{em}$ todos os casos). Esse resultado demonstra que os parâmetros de estrutura estimados se relacionam, e sofrem influência principalmente da abundância dos indivíduos. Estes seguiram a mesma tendência, fato verificado em todas as análises realizadas, sendo também encontrado nos estudos de Oliveira e Amaral (2005) e Nappo (1999).

As famílias mais representativas, considerando a Regeneração Natural Relativa, foram Burseraceae, Marantaceae, Violaceae e Lecythidaceae (Figura 3). A frequência relativa destas foi similar nos anos amostrados. A família Burseraceae foi a mais frequente em todos os anos, sendo a família dominante na maioria dos anos abordados (1998, 1999, 2001 e 2010), o que demonstra a sua importância na área de estudo. Segundo Leitão Filho (1987), Burseraceae e Lecythidaceae estão entre as famílias que comumente apresentam maior número de espécies e indivíduos em florestas tropicais de terra firme na Amazônia brasileira.

Lecythidaceae teve seu valor máximo em 2000, exceto para frequência, que permaneceu constante, atingindo seu ponto máximo nesse ano, quando foi a família mais importante da comunidade, decrescendo nos anos posteriores.

Violaceae teve um acréscimo em 1999 em relação a 1998, porém, decresceu a partir de 2001 até o último ano mensurado. Violaceae e Lecythidaceae apresentaram a mesma tendência da abundância da comunidade, com seu valor máximo em 2000 e decrescendo nos anos seguintes, demonstrando que a maioria das espécies dessas famílias acompanha a tendência da sucessão na área de estudo.

Marantaceae foi a única família de ervas a se destacar na análise estrutural, chegando a ser a dominante em 2007, demonstrando tendência contrária à apresentada pelas demais famílias dominantes, aparentemente beneficiada pela fase sucessional de crescimento. Essa fase sucessional é caracterizada por intensa competição interespecífica por água e nutrientes, onde não há forte radiação que favoreça espécies intolerantes à sombra. Devido à família ser formada por ervas perenes de grandes populações (FORZZA, 2007), a maioria das espécies é bem adaptada a ambientes sombreados, o que explica a tendência crescente de ocupação no sub-bosque com o fechamento das clareiras.

A dinâmica observada na estrutura das diferentes formas de vida está expressa na Figura 4. 


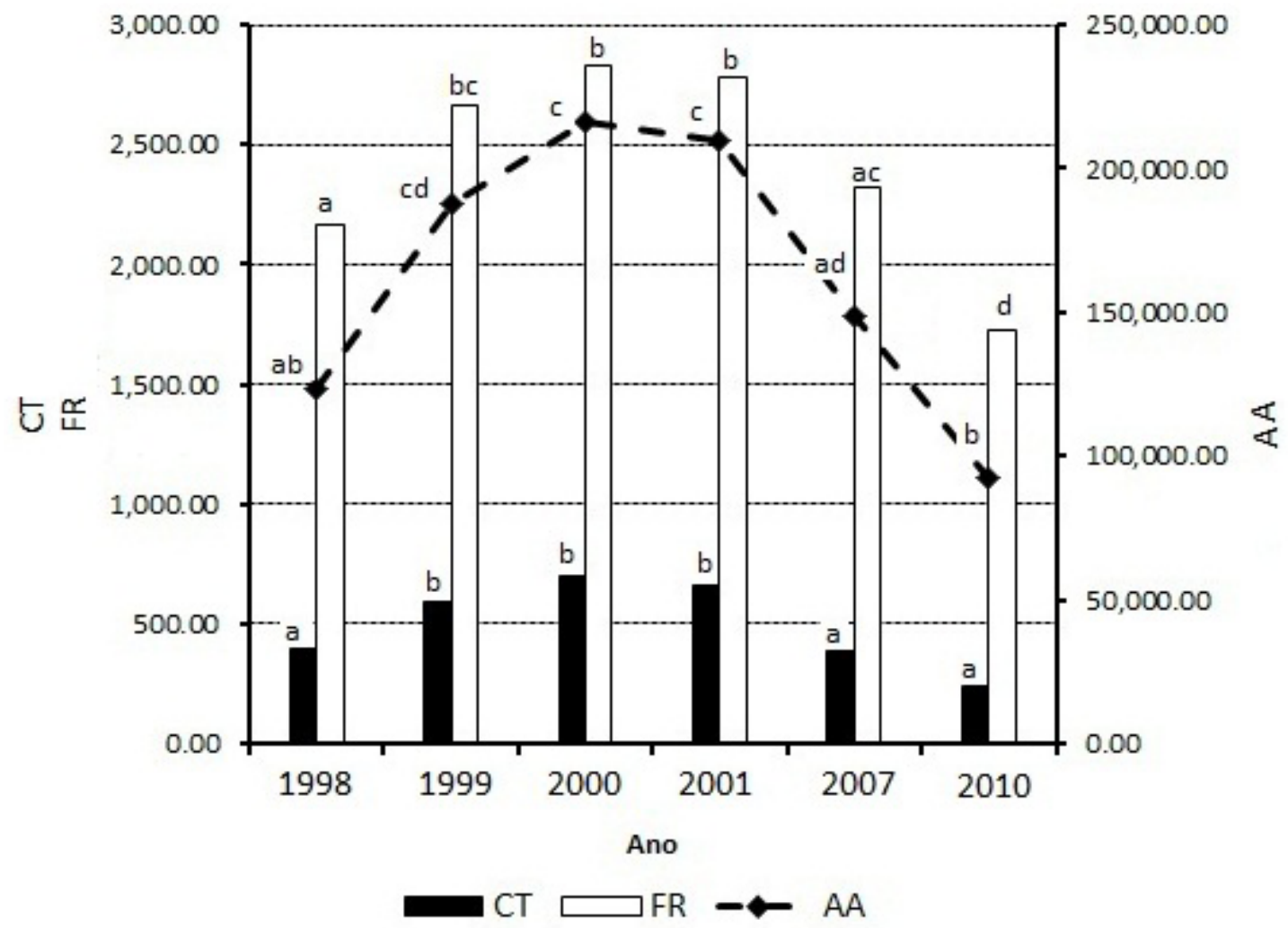

FIGURA 2: Média de Categoria de tamanho (CT, N.ha ${ }^{-1}$ ), frequência (FR) e abundância (AA, N.ha ${ }^{-1}$ ) absolutas, da comunidade em seis medições no período de doze anos em uma amostra de 468 $\mathrm{m}^{2}$ de floresta de terra firme, explorada sob impacto reduzido, em Moju, PA, considerando indivíduos de $\mathrm{HT} \geq 10 \mathrm{~cm}$ a DAP $<5 \mathrm{~cm}$. Anos com letras diferentes são significativamente diferentes de acordo com o Teste de Tukey $(\alpha=0,05)$.

FIGURE 2: Average size classes (CT, N.ha ${ }^{-1}$ ), frequency (FR), and abundance (AA, N.ha ${ }^{-1}$ ) of the vegetation community in six occasions during 12 years, in a $468 \mathrm{~m}^{2}$ sample of terra firme forest logged using techniques of reduced impact logging, in Moju, PA, considering HT $\geq 10 \mathrm{~cm}$ to DBH $<5 \mathrm{~cm}$ individuals. Years with different letters are significantly different according to Tukey Test $(\alpha=0,05)$.

As árvores foram predominantes em todos os anos amostrados, seguidas das ervas e lianas; os arbustos foram mais representativos que as palmeiras até 2001, sendo que as palmeiras foram mais representativas nos anos posteriores.

As árvores tiveram o mesmo comportamento do número de indivíduos e espécies da comunidade em geral, com a abundância, categoria de tamanho e de regeneração natural relativas crescentes até 2000, decrescendo nos anos posteriores. Por se tratar da forma de vida dominante, consequentemente, essa forma de vida influencia a estrutura da comunidade como um todo.

As lianas apresentaram variação nos parâmetros estruturais estimados no decorrer do período analisado. Este comportamento para essa forma de vida é característico em médio prazo nas áreas exploradas (GERWING e UHL, 2003). Contudo, a tendência dessa forma de vida foi contrária ao verificado na comunidade, apresentando valores decrescentes até 2001, e crescendo a partir de 2007, exceto para frequência. É provável que, devido ao caráter heliófilo das espécies dessa forma de vida, a tendência decrescente nos primeiros anos seja devido ao fechamento da clareira, tendendo a estabilização nos anos posteriores.

As ervas mantiveram tendência constante nos quatro primeiros anos amostrados e cresceram em 2007, exceto para a frequência. Como a maioria das espécies herbáceas é bem adaptada a ambientes sombreados, estas foram beneficiadas pelo fechamento das clareiras, acompanhando a tendência da família mais representativa desse grupo (Marantaceae). 

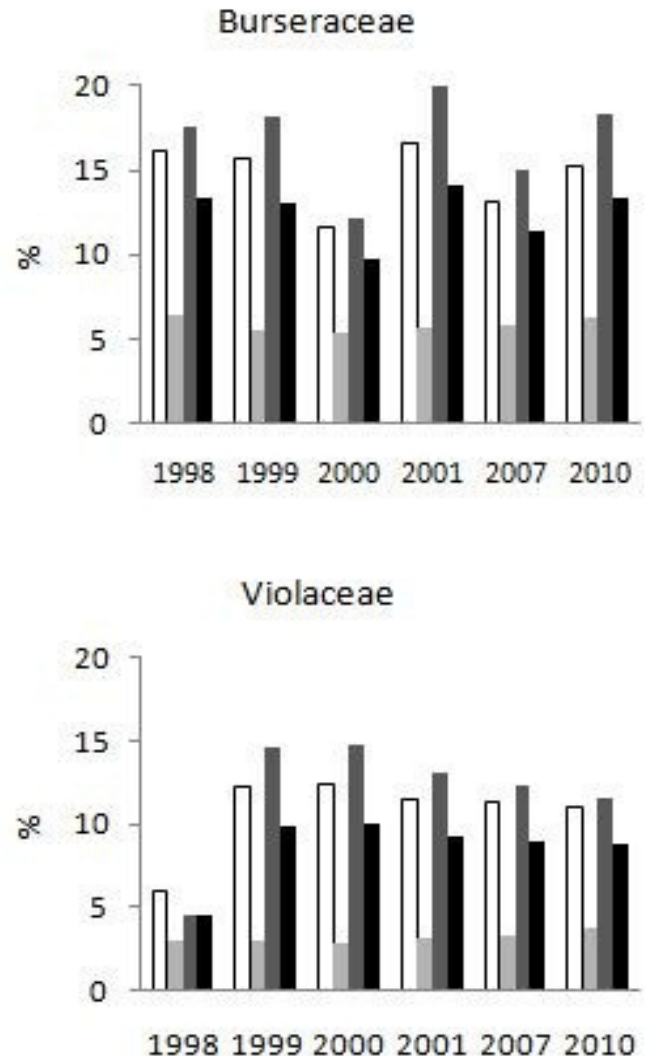

Lecythidaceae

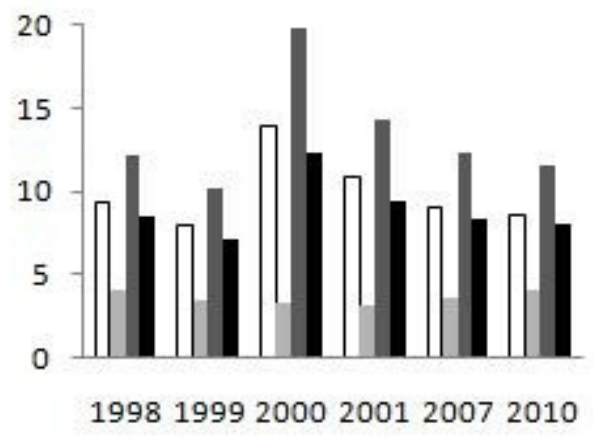

Marantaceae

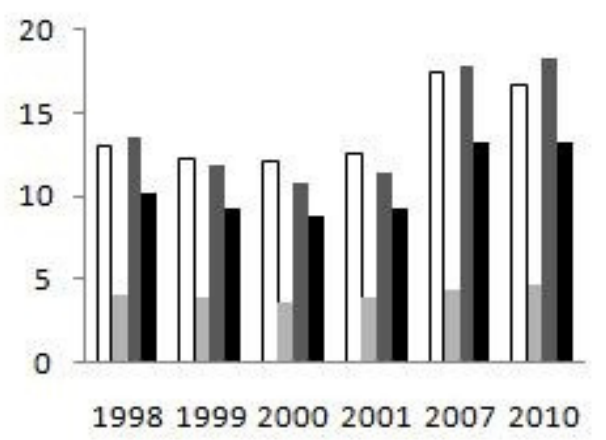

\section{$\square \mathrm{AA}=\mathrm{FR} \quad \mathrm{CT} \mathbf{\mathrm { RN }}$}

FIGURA 3: Abundância (AA), Frequência (FR), Categoria de Tamanho (CT) e Regeneração Natural (RN) Relativas das quatro famílias mais importantes da comunidade, em seis medições no período de 12 anos em $468 \mathrm{~m}^{2}$ de floresta de terra firme, explorada sob impacto reduzido, em Moju, $\mathrm{PA}$, considerando indivíduos de $\mathrm{HT} \geq 10 \mathrm{~cm}$ a DAP $<5 \mathrm{~cm}$.

FIGURE 3: Relative Abundance (AB), Frequency (FR), Size Classes (CT) and Natural Regeneration (RN) of the four most important families in the community, in six occasions during 12 years, in a $468 \mathrm{~m}^{2}$ sample of terra firme forest logged using techniques of reduced impact logging, in Moju, Pará state, considering $\mathrm{HT} \geq 10 \mathrm{~cm}$ to $\mathrm{DAP}<5 \mathrm{~cm}$ individuals.

Os parâmetros relativos estimados para as palmeiras foram crescentes até 2007, com queda em 2010. Salm et al. (2005) afirmam que a ocorrência de palmeiras em áreas de floresta densa é inferior quando comparada com áreas abertas. Ivanauskas et al. (2004), analisando a composição de trechos florestais, encontraram uma representatividade inferior a $2 \%$ para palmeiras em comparação com as demais formas de vida.

A regeneração de palmeiras depende de um conjunto de variáveis ecológicas, que são resultado da dispersão, estabelecimento e resistência das espécies (CORREA-GÓMEZ e VARGAS-RÍOS, 2009). Porém, o número de espécies e indivíduos dessa forma de vida na área permaneceu constante no período estudado (MENDES et al., 2010), resultado semelhante ao encontrado por Jardim e Souza (1996) nas mesmas categorias de tamanho em uma floresta manejada, sendo que os autores atribuíram as mudanças dessa forma de vida a variações florísticas naturais, e não à sucessão.

Os arbustos perderam importância na estrutura da comunidade com o decorrer da sucessão. Essa forma de vida mostrou forte recrutamento em ambiente com maior radiação, indicando forte heliofilia para germinação de sementes (JARDIM e SOUZA, 1996), o que justifica, portanto, a redução da representatividade com o fechamento das 
Árvores

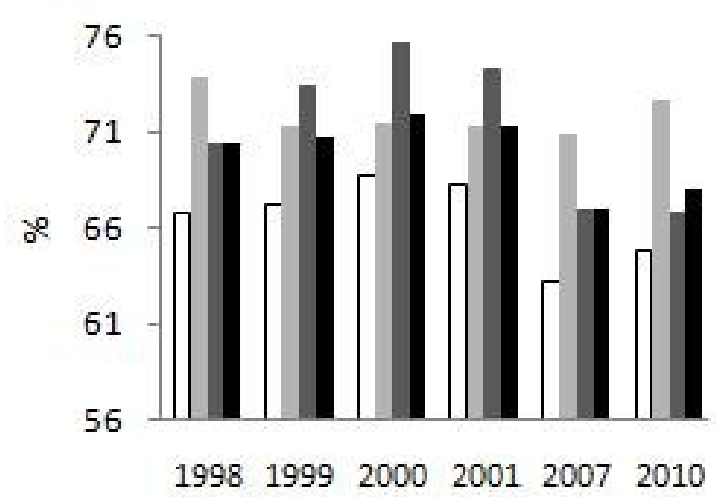

Lianas

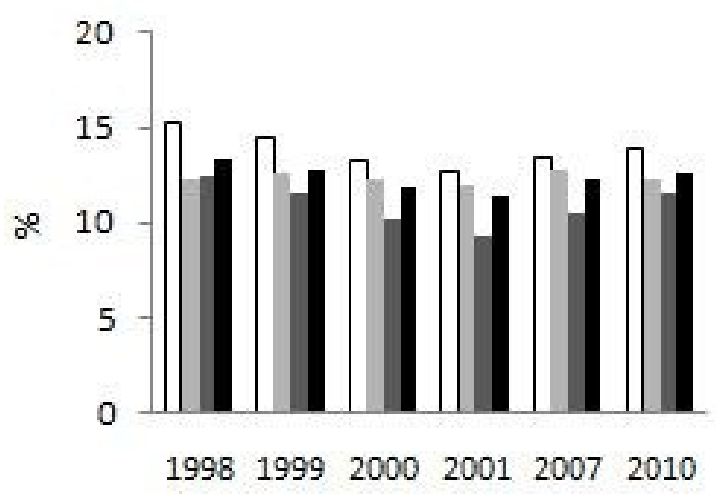

Ervas

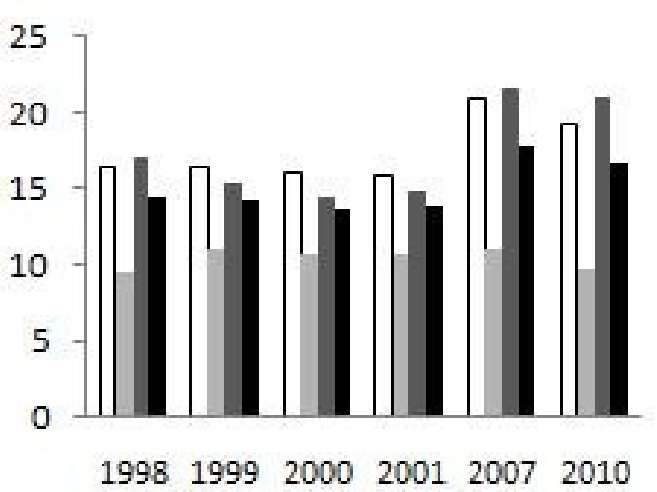

Arbustos

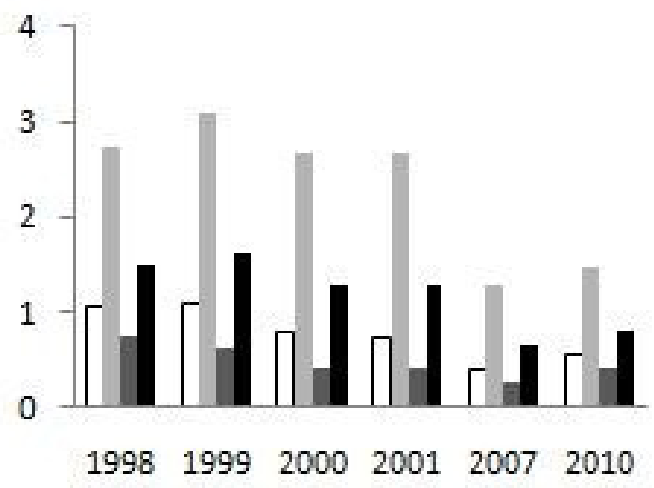

Palmeiras

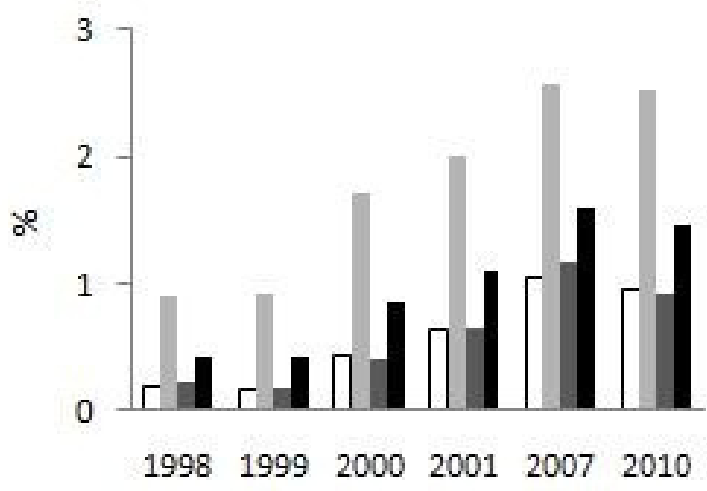

\section{DAA $1 \mathrm{FR}=\mathrm{CT} \square \mathrm{RN}$}

FIGURA 4: Abundância (AB), Frequência (FR), Categoria de Tamanho (CT) e Regeneração Natural Relativa (RN) das formas de vida em seis medições no período de 12 anos em $468 \mathrm{~m}^{2}$ de floresta de terra firme, explorada sob impacto reduzido, em Moju, PA, considerando indivíduos de $\mathrm{HT} \geq 10 \mathrm{~cm}$ a DAP $<5 \mathrm{~cm}$.

FIGURE 4: Relative Abundance (AB), Frequency (FR), Size Classes (CT) and Natural Regeneration (RN) of the life-forms, in six occasions during 12 years, in a $468 \mathrm{~m}^{2}$ sample of terra firme forest logged using techniques of reduced impact logging, in Moju, PA, considering $\mathrm{HT} \geq 10 \mathrm{~cm}$ to $\mathrm{DBH}<5 \mathrm{~cm}$ individuals. 
clareiras na fase de crescimento.

A frequência das três formas de vida dominantes (árvores, ervas e lianas) permaneceu constante nos anos de medição. Isso demonstra que a distribuição das mesmas foi pouco influenciada pela sucessão e fechamento das clareiras. Já para palmeiras e arbustos, a frequência foi influenciada pela sucessão com a mesma tendência dos demais parâmetros estimados.

As 10 espécies mais abundantes da comunidade contribuíram com 48,32\% $\% 3,82$ do total de indivíduos da comunidade (Tabela 1). Dentre estas espécies, Lecythis idatimon, Protium spp. e Eschweilera coriacea são espécies comerciais e potencialmente comerciais, possuindo regeneração assegurada na área, como já demonstraram trabalhos realizados naquela área (KISHI et al., 2000; NASCIMENTO, 2003), caso as condições ambientais permaneçam similares, possibilitando o seu estabelecimento.

As 10 espécies mais frequentes da comunidade estão representadas na Tabela 2. A frequência é um parâmetro que estima a distribuição horizontal das espécies no terreno (HOSOKAWA, 1986). Todavia, esse parâmetro apresenta relação com a abundância das espécies, conforme verificado por Jardim (1990).

A frequência das espécies mais representativas da área se manteve constante no decorrer dos anos. Mesmo Protium pilosum, Protium sp1. e Rinorea guianensis que sofreram reduções nos demais parâmetros estimados em alguma medição, mantiveram essa tendência. Isso demonstra que em relação a esse parâmetro na área amostrada, assim como ocorreu com as famílias e formas de vida mais importantes, a formação de clareiras e o decorrer da sucessão não alteraram a distribuição horizontal das referidas espécies na área.

As espécies mais representativas da Categoria de Tamanho Relativa são representadas na Tabela 3, respondendo em média por $50,33 \% \pm$ 3,05 do total. Observa-se que na categoria de tamanho da regeneração natural relativa, as árvores mais representativas obtiveram maiores valores médios em relação aos outros parâmetros estimados, o que pode ser explicado pela melhor distribuição de indivíduos em todas as categorias de tamanho, ajustando-se melhor à distribuição contínua e decrescente (J-invertido), tendo em vista que as demais formas de vida analisadas por características próprias das espécies, em sua maioria não atingem as categorias de tamanho maiores analisadas.

Segundo Finol (1971), a categoria de tamanho estima a presença das espécies em diferentes estratos em uma floresta. Exceto pelas espécies que não alcançam o estrato médio e superior devido às características genéticas, uma espécie tem seu estabelecimento assegurado na estrutura e composição da comunidade somente quando se encontra representada em todos os estratos, o que é um bom indica-

TABELA 1: Abundância Relativa das 10 espécies principais em seis medições no período de doze anos em uma amostra de $468 \mathrm{~m}^{2}$ de floresta de terra firme, explorada sob impacto reduzido, em Moju, $\mathrm{PA}$, considerando indivíduos de $\mathrm{HT} \geq 10 \mathrm{~cm}$ a DAP $<5 \mathrm{~cm}$.

TABLE 1: Relative Abundance of the 10 main species in six occasions during 12 years, in a $468 \mathrm{~m}^{2}$ sample of terra firme forest logged using techniques of reduced impact logging, in Moju, Pará state, considering $\mathrm{HT} \geq 10 \mathrm{~cm}$ to $\mathrm{DBH}<5 \mathrm{~cm}$ individuals.

\begin{tabular}{lcccccccc}
\hline Nome científico & Família & FV & 1998 & 1999 & 2000 & 2001 & 2007 & 2010 \\
\hline Eschweilera coriacea (DC.) S.A.Mori & Lecythidaceae & AR & 3,20 & 2,53 & 3,55 & 3,35 & 2,66 & 3,12 \\
Lecythis idatimon Aubl. & Lecythidaceae & AR & 5,54 & 4,94 & 10,01 & 7,13 & 5,83 & 4,90 \\
Protium pilosum (Cuatrec.) Daly & Burseraceae & AR & 3,72 & 2,75 & 2,38 & 3,78 & 7,10 & 7,62 \\
Protium sp1. & Burseraceae & AR & 5,22 & 4,84 & 4,17 & 5,44 & 2,43 & 4,39 \\
Rinorea flavescens (Aubl.) Kuntze & Violaceae & AR & 2,34 & 2,61 & 2,93 & 3,10 & 4,54 & 4,16 \\
Rinorea guianensis Aubl & Violaceae & AR & 3,55 & 9,41 & 9,28 & 8,13 & 6,53 & 6,56 \\
Monotagma sp1. & Marantaceae & ER & 7,54 & 6,30 & 6,13 & 6,33 & 8,97 & 9,08 \\
Monotagma sp2. & Marantaceae & ER & 4,39 & 4,90 & 4,94 & 4,97 & 6,97 & 6,61 \\
Bauhinia spp. & Fabaceae & LI & 5,02 & 4,16 & 3,77 & 3,44 & 3,30 & 3,81 \\
Davilla cuspidulata & Dilleniaceae & LI & 2,49 & 2,59 & 2,32 & 2,28 & 2,90 & 2,96 \\
\hline
\end{tabular}

Em que: $\mathrm{FV}$ = forma de vida: $\mathrm{AR}$ = árvores; $\mathrm{ER}=$ ervas; e $\mathrm{LI}=$ lianas. 
TABELA 2: Frequência absoluta das 10 espécies principais em seis medições no período de doze anos em uma amostra de $468 \mathrm{~m}^{2}$ de floresta de terra firme, explorada sob impacto reduzido, em Moju, $\mathrm{PA}$, considerando indivíduos com $\mathrm{HT} \geq 10 \mathrm{~cm}$ e DAP $<5 \mathrm{~cm}$.

TABLE 2: Frequency of the 10 main species in six occasions during 12 years, in a $468 \mathrm{~m}^{2}$ sample of terra firme forest logged using techniques of reduced impact logging, in Moju, PA, considering HT $\geq 10 \mathrm{~cm}$ to $\mathrm{DBH}<5 \mathrm{~cm}$ individuals.

\begin{tabular}{lcccccccc}
\hline Nome científico & Família & FV & 1998 & 1999 & 2000 & 2001 & 2007 & 2010 \\
\hline Protium sp1. & Burseraceae & AR & 54,7 & 58,1 & 56,4 & 56,4 & 58,0 & 55,6 \\
Eschweilera coriacea (DC.) S.A.Mori & Lecythidaceae & AR & 64,1 & 70,9 & 77,8 & 72,6 & 63,2 & 46,1 \\
Lecythis idatimon Aubl. & Lecythidaceae & AR & 70,9 & 74,6 & 82,9 & 82,0 & 78,6 & 58,1 \\
Pouteria gongrijpii Eyma & Sapotaceae & AR & 70,9 & 70,9 & 66,7 & 66,7 & 50,4 & 39,3 \\
Rinorea flavescens (Aubl.) Kuntze & Violaceae & AR & 44,4 & 58,1 & 60,7 & 63,2 & 64,1 & 53,0 \\
Rinorea guianensis Aubl. & Violaceae & AR & 68,4 & 76,9 & 83,8 & 84,6 & 82,0 & 73,5 \\
Monotagma sp1. & Marantaceae & ER & 65,8 & 66,7 & 68,4 & 68,4 & 65,0 & 58,1 \\
Monotagma sp2. & Marantaceae & ER & 73,5 & 82,0 & 86,3 & 84,6 & 83,8 & 65,1 \\
Adenocalymma sp. & Bignoniaceae & LI & 70,9 & 79,5 & 83,6 & 81,20 & 72,65 & 0,85 \\
Bauhinia spp. & Fabaceae & LI & 71,8 & 73,5 & 73,5 & 73,5 & 65,8 & 53,8 \\
\hline
\end{tabular}

Em que: $\mathrm{FV}$ = forma de vida: $\mathrm{AR}=$ árvores; $\mathrm{ER}=$ ervas; e $\mathrm{LI}=$ lianas.

TABELA 3: Categoria de Tamanho Relativa das 10 espécies principais em seis medições no período de doze anos em uma amostra de $468 \mathrm{~m}^{2}$ de floresta de terra firme, explorada sob impacto reduzido, em Moju, PA, considerando indivíduos de $\mathrm{HT} \geq 10 \mathrm{~cm}$ a DAP $<5 \mathrm{~cm}$.

TABLE 3: Relative Size Classes of the 10 principal species in six occasions during 12 years, in $468 \mathrm{~m}^{2}$ of terra firme forest logged using techniques of reduced impact logging, in Moju, PA, considering $\mathrm{HT} \geq 10 \mathrm{~cm}$ to DAP $<5 \mathrm{~cm}$ individuals.

\begin{tabular}{lcccccccc}
\hline Nome científico & Família & FV & 1998 & 1999 & 2000 & 2001 & 2007 & 2010 \\
\hline Protium pilosum (Cuatrec.) Daly & Burseraceae & AR & 2,87 & 2,04 & 1,68 & 3,41 & 7,69 & 8,13 \\
Protium sp1. & Burseraceae & AR & 5,74 & 5,40 & 4,54 & 6,17 & 2,34 & 5,05 \\
Tetragastris altissima (Aubl.) Swart & Burseraceae & AR & 3,59 & 4,37 & 3,08 & 3,75 & 1,03 & 0,54 \\
Eschweilera coriacea (DC.) S.A.Mori & Lecythidaceae & AR & 3,49 & 2,69 & 3,95 & 3,55 & 2,74 & 3,39 \\
Lecythis idatimon Aubl. & Lecythidaceae & AR & 6,34 & 5,67 & 11,98 & 8,38 & 6,91 & 5,73 \\
Rinorea flavescens (Aubl.) Kuntze & Violaceae & AR & 2,31 & 2,68 & 3,11 & 3,30 & 5,06 & 4,51 \\
Rinorea guianensis Aubl. & Violaceae & AR & 2,79 & 10,38 & 10,21 & 8,71 & 6,47 & 6,49 \\
Monotagma sp1. & Marantaceae & ER & 7,59 & 5,65 & 5,17 & 5,48 & 8,02 & 8,73 \\
Monotagma sp2. & Marantaceae & ER & 4,62 & 5,38 & 5,21 & 5,31 & 8,15 & 7,64 \\
Bauhinia spp. & Fabaceae & LI & 5,30 & 4,11 & 3,66 & 3,12 & 2,85 & 3,74 \\
\hline
\end{tabular}

Em que: $\mathrm{FV}$ = forma de vida: $\mathrm{AR}$ = árvores; $\mathrm{ER}=$ ervas; e $\mathrm{LI}=$ lianas.

dor da perpetuidade das espécies que se destacaram neste parâmetro na área, caso as condições ambientais permaneçam similares as dos anos amostrados.

As espécies com distribuição de tamanho em forma de J-invertido são adaptadas às condições encontradas abaixo do dossel, com o recrutamento de indivíduos de uma categoria inferior para outras maiores ocorrendo de forma contínua, pois o crescimento dos indivíduos é constante nas condições de sub-bosque (MEIRA NETO e MARTINS 2003).

A presença de indivíduos nas categorias de tamanho maiores, associada à distribuição contínua nas demais categorias, pode significar estabelecimento da espécie na área, o que demonstra que as espécies mencionadas foram favorecidas durante $o$ fechamento do dossel decorrente da sucessão natural da floresta (JARDIM et al. 2007; SILVA et al. 2004; NUNES et al. 2003; MORY e JARDIM 2001). 
Duguetia flagellaris Huber., Protium sp1., Protium pilosum (Cuatrec.) Daly, Protium robustum (Swart) D. M. Porter, Lecythis idatimon Aublt., Pouteria gongrijpii (Eyma) Aubrév. e Rinorea guianensis Aubl., foram registradas em todas as categorias de tamanho abordadas em seis medições. Protium paraense Cuatrec, Pourouma guianensis Aubl., apesar de não apresentarem destaque nos parâmetros estimados, estiveram presentes em todas as categorias de tamanho abordadas em todos os anos do presente estudo.

Licania lata J. F. Macbr., Saccoglottis sp., Inga gracilifolia Ducke, Inga sp1., Brosimum guianense Huber ex Ducke, Virola cuspidata Warb., Sterculia speciosa K. Schum e Rinorea flavescens Kuntze não apresentavam indivíduos nas categorias de tamanho superiores nos primeiros anos de medição e não se destacaram nos parâmetros estimados. Entretanto, nos últimos anos da amostragem, a distribuição dessas espécies foi contínua nas categorias de tamanho, demonstrando que sua importância foi crescente na comunidade.

As espécies mais importantes segundo a
Regeneração Natural Relativa estão representadas na Tabela 4 , totalizando $59,93 \% \pm 1,92$. Isso sugere que essas espécies devem permanecer na área por um longo período de tempo em condições ambientais similares, sendo espécies fundamentais para a estrutura da comunidade estudada.

Considerando as espécies mais importantes da comunidade nos parâmetros estimados, assim como ocorreu com a frequência e a abundância, as árvores foram mais representativas no parâmetro Regeneração Natural Relativa da comunidade. Entretanto, nos anos de 1998, 2007 e 2010, a espécie mais importante para esse parâmetro foi do gênero Monotagma, demonstrando a importância desta erva na área estudada.

As lianas representaram a terceira forma de vida mais importante do povoamento, possuindo representantes entre as espécies de maior Regeneração Natural Relativa em todos os anos de medição. Os indivíduos escandentes são formados em sua maioria por espécies dominantes de clareira e fases iniciais de sucessão (GERWING e FARIAS, 2003). Porém, Gerwing (2003) classifi-

TABELA 4: Regeneração Natural Relativa das 20 espécies principais em seis medições no período de doze anos em uma amostra de $468 \mathrm{~m}^{2}$ de floresta de terra firme, explorada sob impacto reduzido, em Moju, PA, considerando indivíduos de $\mathrm{HT} \geq 10 \mathrm{~cm}$ a DAP $<5 \mathrm{~cm}$.

TABLE 4: Relative Natural Regeneration of the 20 principal species in six occasions during 12 years, in $468 \mathrm{~m}^{2}$ of terra firme forest, under reduced impact logging, in Moju, Pará state, considering $\mathrm{HT} \geq 10 \mathrm{~cm}$ to $\mathrm{DAP}<5 \mathrm{~cm}$ individuals.

\begin{tabular}{|c|c|c|c|c|c|c|c|c|}
\hline Nome científico & Família & FV & 1998 & 1999 & 2000 & 2001 & 2007 & 2010 \\
\hline Duguetia flagellaris Huber & Annonaceae & AR & 2,51 & 1,91 & 1,75 & 1,71 & 2,04 & 2,46 \\
\hline Protium pilosum (Cuatrec.) Daly & Burseraceae & AR & 3,04 & 2,32 & 2,02 & 3,07 & 5,77 & 6,32 \\
\hline Protium sp1. & Burseraceae & AR & 4,8 & 4,43 & 3,85 & 4,83 & 2,36 & 4,09 \\
\hline Tetragastris altissima (Aubl.) Swart & Burseraceae & AR & 2,34 & 2,77 & 1,98 & 2,37 & 0,78 & 0,51 \\
\hline Eschweilera coriacea (DC.) S.A.Mori & Lecythidaceae & AR & 3,22 & 2,63 & 3,42 & 3,17 & 2,7 & 3,06 \\
\hline Lecythis idatimon Aubl. & Lecythidaceae & AR & 5,05 & 4,47 & 8,31 & 6,15 & 5,36 & 4,66 \\
\hline Inga sp1. & Fabaceae & AR & 1,94 & 2,2 & 2,13 & 2,09 & 1,59 & 1,64 \\
\hline Pouteria gongrijpii Eyma & Sapotaceae & AR & 3,34 & 2,43 & 2,11 & 2,07 & 1,77 & 1,82 \\
\hline Rinorea flavescens (Aubl.) Kuntze & Violaceae & AR & 2,23 & 2,49 & 2,73 & 2,89 & 4,11 & 3,91 \\
\hline Rinorea guianensis Aubl. & Violaceae & AR & 3,16 & 7,56 & 7,48 & 6,63 & 5,5 & 5,77 \\
\hline Monotagma sp1. & Marantaceae & ER & 6,05 & 4,82 & 4,57 & 4,75 & 6,59 & 7,06 \\
\hline Monotagma sp2. & Marantaceae & ER & 4,13 & 4,45 & 4,4 & 4,44 & 6,23 & 6,02 \\
\hline Adenocalyma spp. & Bignoniaceae & LI & 2,9 & 2,59 & 2,49 & 2,43 & 2,78 & 3,13 \\
\hline Bauhinia spp. & Fabaceae & LI & 4,54 & 3,68 & 3,34 & 3,07 & 2,99 & 3,55 \\
\hline Davilla cuspidulata Mart. ex Eichler & Dilleniaceae & LI & 2,31 & 2,42 & 2,18 & 2,17 & 2,68 & 2,74 \\
\hline
\end{tabular}

Em que: $\mathrm{FV}$ = forma de vida: $\mathrm{AR}=$ árvores; $\mathrm{ER}=$ ervas; e $\mathrm{LI}=$ lianas. 
ca Memora schomburgkii, atualmente denominada de Adenocalymma schomburgkii, e Bauhinia guianensis como espécies de sucessão avançada, sendo que o gênero de ambas as espécies é o mais representativo da forma de vida.

As demais formas de vida foram pouco representativas neste parâmetro de estrutura na comunidade. As cinco espécies mais representativas, segundo este último parâmetro no decorrer das seis medições, foram Protium pilosum, Protium sp1., Lecythis idatimon, Rinorea guianensis e Monotagma sp1. e Monotagma sp2. Observa-se que a Categoria de Tamanho Relativa é proporcional à abundância da espécie e, consequentemente, a Regeneração Natural Relativa é influenciada pelo número de indivíduos da espécie.

Quando comparados os parâmetros estruturais de Lecythis idatimon, Rinorea guianensis e Monotagma spp.,verificou-se que essas espécies foram tão dominantes que influenciaram a estrutura das famílias a que pertencem, Lecythidaceae, Violaceae e Marantaceae, respectivamente. No caso de Monotagma spp., a influência estendeu-se também às ervas.

Algumas espécies, como Protium pilosum e Monotagma spp., apresentaram valores crescentes nos últimos anos para os parâmetros estimados, mesmo com a tendência de redução no número de indivíduos na comunidade como um todo, indicando que são espécies favorecidas pela fase sucessional de crescimento em que se encontra a área.

Lecythis idatimon e Rinorea guianensis aumentaram sua abundância na fase de clareira e reduziram na fase de crescimento, de acordo com o padrão geral da comunidade em questão. Porém, mesmo com essa redução, continuam sendo espécies altamente representativas na comunidade segundo os parâmetros estimados.

Monotagma sp1. ocorreu na CT3 $(130 \mathrm{~cm}$ $<$ HT e DAP < $2 \mathrm{~cm}$ ) somente em 1998 e 2010, e Monotagma sp2. em 1998, 2007 e 2010, e, por serem ervas, não ocorreram na CT4 $(2 \mathrm{~cm} \leq \mathrm{DAP}$ $<5 \mathrm{~cm}$ ). Mesmo assim essas espécies destacam-se na categoria de tamanho relativa e, consequentemente, na regeneração natural relativa.

Espécies como Duguetia flagellaris, Protium paraense, Protium robustum, Pourouma guianensis, Eschweilera coriacea e Pouteria gongrijpii, que apresentaram distribuição regular nas categorias de tamanho consideradas, não se destacaram no parâmetro estimados por possuírem abundância inferior às espécies mais importantes na regeneração natural relativa. Este fato foi observado também por Oliveira e Amaral (2005).

\section{CONCLUSÃO}

A abundância, a categoria de tamanho e a regeneração natural relativas das espécies do sub-bosque sofreram alterações no período estudado. Entretanto, a frequência das espécies, famílias e formas de vida mais representativas não sofreram alterações marcantes. Isto pode indicar que o período avaliado (12 anos) ainda não é suficiente para concluir sobre as mudanças na estrutura do sub-bosque, visto que não foi observada tendência à estabilidade para a maioria dos parâmetros analisados.

A dinâmica das diferentes formas de vida demonstrou ser dependente da formação de clareiras e, consequentemente, da fase sucessional da área. A abertura de clareiras possibilitou o estabelecimento de regeneração de espécies comerciais e potencialmente comerciais, indicando que essas espécies, até os 12 anos após a exploração, não necessitam de condução para garantir a regeneração para futuros cortes.

\section{REFERÊNCIAS BIBLIOGRÁFICAS}

ALVES, J. C. Z. O.; MIRANDA, I. de S. Análise da estrutura de comunidades arbóreas de uma floresta amazônica de terra firme aplicada ao manejo florestal. Acta Amazonica, v. 38, n. 4, p. 657- 666, 2008.

APG III. An update of the Angiosperm Phylogeny Group classification for the orders and families of flowering plants: APG III. Botanical Journal of the Linnean Society, v. 161, p. 105-121, 2009.

BLOOR, J. M. G.; GRUBB P. J. Growth and mortality in high and low light: trends among 15 shade-tolerant tropical rain forest tree species. Journal of Ecology, v. 91, p. 77-85. 2003. CORREA-GÓMEZ, D. F.; VARGAS-RÍOS, O. Regeneración de palmas en bosques nativos y plantaciones del Santuario De Fauna Y Flora Otún - Quimbaya (Risaralda, Colombia). Caldasia, v. 31, n. 2, p. 195-212, 2009.

FELFILI, J. M. et al. Desenvolvimento inicial de espécies de Mata de Galeria. p. 779-811. In: RIBEIRO, J. F.; FONSECA, C. E. L.; SOUSASILVA J. C. Cerrado: caracterização e recuperação de matas de galeria. Planaltina: Embrapa Cerrados. 2001.

FINOL, U. H. Nuevos parámetros a considerarse 
en el análisis estructural de las selva vírgenes tropicales. Revista Forestal Venezolana, v. 14, n. 21, p. 29-42, 1971.

FORZZA, R. C. Flora da Reserva Ducke, Amazonas, Brasil: Marantaceae. Rodriguésia, v. 58, n. 3, p. 533-543. 2007.

GERWING, J. J. A diversidade de histórias de vida natural entre seis espécies de cipó do dossel em uma floresta nativa na Amazônia Oriental. In: VIDAL, E.; GERWING, J. J. (Org.). Ecologia e manejo de cipós na Amazônia Oriental. Belém: Imazon, 2003. p. 92-55.

GERWING, J. J.; FARIAS, D. Estimativa de biomassa total a partir da abundância de cipós e estatura da floresta na Amazônia Oriental. In: VIDAL, E.; GERWING, J. J. (Org.). Ecologia e manejo de cipós na Amazônia Oriental. Belém: Imazon, 2003. p. 119-91.

GERWING, J. J.; UHL, C. Impactos do corte de cipós antes da exploração de madeira na sua regeneração em clareiras de exploração na Amazônia Oriental. In: VIDAL, E.; GERWING, J. J. (Org.). Ecologia e manejo de cipós na Amazônia Oriental. Belém: Imazon, 2003. p. 35-55.

HOSOKAWA, R. T. Manejo e economia de florestas. Roma, FAO, 1986. p. 125.

IPNI. The International Plant Names Index (disponível em: http://www.ipni.org/). Acesso: 16/06/2010.

IVANAUSKAS, N. M.; MONTEIRO, R.; RODRIGUES, R. R. Composição florística de trechos florestais na borda sul-amazônica. Acta Amazonica. v. 34, n. 3. p. 399-413, 2004.

JARDIM, F. C. da S. Relação entre a abundância e a frequência na floresta equatorial de terra firme. Acta Amazonica, v. 20, p. 185-192. 1990.

JARDIM, F. C. da S.; HOSOKAWA, R. T. Estrutura da floresta equatorial úmida da estação experimental de silvicultura tropical do INPA. Acta Amazonica, v. 16/17 (nº́nico), p. 411-508, 1987.

JARDIM, F. C. da S.; SERRÃO, D. R.; NEMER, T. C. Efeito de diferentes tamanhos de clareiras sobre o crescimento e a mortalidade de espécies arbóreas, em Moju - PA. Acta Amazonica, v. 37, p. 37-48. 2007

JARDIM, F. C. S., SOUZA, A. L. Dinâmica da vegetação herbáceo-arbustiva com DAP menor que 5,0 cm na Estação Experimental de Silvicultura Tropical do INPA, Manaus-AM. Revista Árvore , v.20, p. 433-442, 1996.

KISHI, I. A. S., et al. Análise estrutural de uma floresta de terra firme explorada seletivamente,
Moju-PA In: SEMINÁRIO DE INICIAÇÃO CIENTÍFICA DA FCAP, 10; SEMINÁRIO DE INICIAÇÃO CIENTÍFICA DA EMBRAPA AMAZÔNIA ORIENTAL, 4., 2000, Belém. Resumos. Belém: SDI/FCAP, v.1, p. 270- 272, 2000.

KOZERA, C.; RODRIGUES, R. R., DITTRICH, V. A. de O. Composição florística do sub-bosque de uma Floresta Ombrófila Densa Montana, Morretes, PR, Brasil. Floresta, Curitiba, v. 39, n. 2, p. 323334. 2009.

LEITÃO FILHO, H. de F. Considerações sobre a florística de florestas tropicais e sub-tropicais do Brasil. IPEF, n. 35, p. 41-46, 1987.

LIMA FILHO, D. de A. et al. Regeneração natural de três hectares de floresta ombrófila densa de terra firme na região do Rio Urucu-AM, Brasil. Acta Amazonica, v. 31 n. 4, p. 555-570, 2002.

LOPES, J. do C. A. et al. Efeito da exploração florestal nas populações de mudas em uma floresta tropical úmida no município de Mojú, PA. In: SILVA, J. N. M.; CARVALHO, J. O. P.; YARED, J. A. G (Ed.). A silvicultura na Amazônia Oriental: contribuições do projeto Embrapa/DFID. Belém: Embrapa Amazônia Oriental: DFID, 2001. p. 203226.

MEIRA NETO, J. A. A.; MARTINS, F. R. Estrutura do sub-bosque herbáceo-arbustivo da Mata da Silvicultura, uma floresta estacional semidecidual no município de Viçosa-MG. Revista Árvore, v. 27, p. 459-471. 2003.

MENDES, F. da S. et al. Dinâmica da composição florística do sub-bosque em floresta tropical manejada, no município de Moju, estado do Pará, Brasil. Revista de Ciências Agrárias, v. 55, n 2, p. 117-123. 2012.

MORY, A. de M.; JARDIM, F. C. da S. Comportamento de Goupia glabra Aubl. (Cupiúba) em diferentes níveis de desbastes por anelamento em plantas naturais. Revista de Ciências Agrárias, v. 36, p. 55-66. 2001.

NAPPO, M. E. Inventário florístico e estrutural da regeneração natural no sub-bosque de povoamentos homogêneos de Mimosa scabrella Bentham, implantados em áreas mineradas, em Poços de Caldas, Minas Gerais. 1999. 123 f. Dissertação (Mestrado em Ciência Florestal). Universidade Federal de Viçosa, Viçosa, 1999.

NASCIMENTO, Z. P. D. Dinâmica populacional de Lecythis idatimon Aublet após exploração florestal seletiva em uma floresta tropical de terra-firme. 2003. 66 f. Dissertação (Mestrado em 
Ciências Florestais) - Universidade Federal Rural da Amazônia, Belém, 2003.

NUNES, Y. R. F. et al. Variações da fisionomia, diversidade e composição de guildas da comunidade arbórea em um fragmento de floresta semidecidual em Lavras, MG. Acta botanica brasilica. v. 17, n. 2, p. 213-229. 2003.

OLIVEIRA, A. N. de. et al. Composição e diversidade florístico-estrutural de um hectare de floresta densa de terra firme na Amazônia Central, Amazonas, Brasil. Acta Amazonica, v. 38, n. 4, p. 627-642, 2008.

OLIVEIRA, A. N. de; AMARAL, I. L. do. Aspectos florísticos, fitossociológicos e ecológicos de um sub-bosque de terra firme na Amazônia Central, Amazonas, Brasil. Acta Amazonica. v. 35, n. 1. p. 1-16, 2005.

PIMENTEL-GOMES, F; GARCIA, C. H. Estatística aplicada a experimentos agronômicos e florestais: exposições com exemplos e orientações para uso de aplicativos. Piracicaba: FEALQ, 2002. 309 p.

PIRES-O'BRIEN, M. J.; O'BRIEN, C. M. Ecologia e modelamento de florestas tropicais. Belém: FCAP., 1995. p. 400.

SALLES, J. C.; SCHIAVINI, I. Estrutura e composição do estrato de regeneração em um fragmentoflorestal urbano: implicações para a dinâmica e a conservação da comunidade arbórea. Acta botanica brasilica, v. 21, n.1, p. 223-233, 2007.

SALM, R., JALLES-FILHO, E.; SCHUCKPAIM, C. A model for the importance of large arborescent palms in the dynamics of seasonally- dry Amazonian forests. Biota Neotropica. v. 3, n.1, 2005.

SANTOS, J. U. M. dos. et al. Vegetação da Área de Proteção Ambiental Jabotitiua-Jatium. Município de Viseu, Pará, Brasil. Acta Amazonica. v. 33, n. 3. p. 431-444, 2003.

SANTOS, P. L. dos. et al. Levantamento semidetalhado dos solos e avaliação da aptidão agrícola das terras para culturas de dendê e seringueira. Projeto Moju, Pará: Relatório técnico. Rio de Janeiro: Embrapa / SNLCS. 1985. p. 192. SILVA, J. A. et al. Caracterização florística, fitossociológica e regeneração natural do subbosque da Reserva Genética Florestal Tamanduá, DF. Ciência Florestal, v. 14, n. 1, p. 121-132. 2004. SILVA, K. E. da; MATOS, F. D. de A.; FERREIRA, M. M. Composição florística e fitossociologia de espécies arbóreas do Parque Fenológico da Embrapa Amazônia Ocidental. Acta Amazonica. v. 38, n. 2, p. 213-222, 2008.

SILVA, S. M. A. da. et al. Impacto da exploração madeireira em uma floresta de terra firme no município de Mojú, estado do Pará. In: SILVA, J. N. M.; CARVALHO, J. O. P.; YARED, J. A. G (Ed.). A silvicultura na Amazônia Oriental: contribuições do projeto Embrapa/DFID. Belém: Embrapa Amazônia Oriental: DFID, 2001. p. 309-323.

VELOSO, H. P.; RANGEL FILHO, A. L. R.; LIMA, J. C. A. Classificação da vegetação brasileira adaptada a um sistema universal. Rio de Janeiro: IBGE, Departamento de Recursos Naturais e Estudos Ambientais, 1991. p. 124.

WHITMORE, T. C. Tropical rain forest of the Far East. $2^{\text {nd }}$ ed. Oxford: Clarendon Press, 1984, 352 p. 